\title{
Cardiac involvement in Wegener's granulomatosis resistant to induction therapy
}

\author{
Tomasz Miszalski-Jamka • Wojciech Szczeklik • Barbara Sokołowska • \\ Karol Miszalski-Jamka • Krzysztof Karwat • Gabriel Grządziel • Wojciech Mazur • \\ Dean J. Kereiakes • Jacek Musial
}

Received: 13 February 2011 /Revised: 12 May 2011 / Accepted: 31 May 2011 /Published online: 23 July 2011

(C) The Author(s) 2011. This article is published with open access at Springerlink.com

\begin{abstract}
Objectives The aim of the study was to assess cardiac involvement in patients with Wegener's granulomatosis (WG), who failed to achieve remission following $>6$ months induction therapy for life or organ threatening disease. Methods Eleven WG patients (eight males, mean age $47 \pm$ 13 years), who failed to achieve remission despite $>6$ months induction therapy, underwent transthoracic echocardiography (TTE) and cardiac magnetic resonance (CMR).

Results Cardiac involvement was present in $9(82 \%)$ patients. Regional wall motion abnormalities were found in two individuals, but none had left ventricular (LV) ejection fraction $<50 \%$. Nine patients had late gadolinium enhancement (LGE) lesions involving LV myocardium and right ventricle free wall was involved in four patients. LGE lesions were found in subepicardial, midwall and subendocardial LV myocardial layers. CMR revealed myocarditis in
\end{abstract}

T. Miszalski-Jamka $(\bowtie) \cdot$ K. Karwat $\cdot$ G. Grządziel

Center for Diagnosis, Prevention and Telemedicine,

John Paul II Hospital,

ul. Prądnicka 80,

31-202 Kraków, Poland

e-mail: miszalt@mp.pl

T. Miszalski-Jamka $\cdot$ W. Szczeklik $\cdot$ B. Sokołowska $\cdot$ J. Musiał Department of Internal Medicine,

Jagiellonian University Medical College,

Krakow, Poland

K. Miszalski-Jamka

1st Department of Cardiology, Silesian Centre for Heart Disease,

Zabrze, Poland

W. Mazur · D. J. Kereiakes

The Christ Hospital Heart and Vascular Center/The Lindner

Center for Research and Education,

Cincinnati, OH, USA six patients. Patients with myocarditis had a higher number of LV segments with LGE $(5.2 \pm 3.4$ vs $1.0 \pm 1.2$, $p=0.03$ ) and more frequent diastolic dysfunction by TTE ( 5 vs $0, p=0.02$ ) than those without. Pericardial effusion was observed in five patients, while localized pericardial thickening in six patients.

Conclusions In WG resistant to $>6$ months induction therapy cardiac involvement is frequent and is characterized by foci of LGE lesions and signs of myocardial inflammatory process.

Keywords Wegener's granulomatosis · Cardiac magnetic resonance $\cdot$ Echocardiography $\cdot$ Myocarditis $\cdot$ Remission

\section{Introduction}

Wegener's granulomatosis (WG) is characterized by systemic necrotizing vascultis and granuloma formation which most often affects the respiratory tract and kidneys [1]. WG is associated with a cytoplasmic pattern of antineutrophil cytoplasmic antibodies (cANCA), with specificity against proteinase-3. Although clinically evident cardiac manifestations are rare, heart involvement is not uncommon and varies substantially depending on disease activity and diagnostic modality $[1,2]$. A wide spectrum of cardiac abnormalities including pericarditis, valvular lesions, coronary arteritis, myocarditis and cardiac rhythm disorders were described [3]. Cardiac involvement evaluated by imaging has only been described in a few studies and most cardiac abnormalities have been described in case reports $[2,4,5]$. Recently, cardiac magnetic resonance (CMR) has emerged as a novel technique providing comprehensive, accurate assessment of myocardial function and structure including detection of myocardial inflammation in patients 
suspected of acute and chronic myocarditis [6-8]. To date, CMR evaluation of cardiac involvement in WG has been limited to a single study and several case reports [9-11].

Cyclophosphamide and corticosteroids, which are predominately used for remission induction in life or organ threatening WG, lead to clinical remission in the majority of patients after 6 months of therapy [12]. Subsequent treatment in patients who fail to achieve remission is poorly defined and is based predominately on disease activity assessment. In those patients no data exists regarding cardiac involvement including signs of myocarditis. Therefore the purpose of the present study was to evaluate cardiac involvement by CMR in patients with WG who failed to achieve remission despite $>6$ months induction therapy for life or organ threatening disease (Fig. 1).

\section{Methods}

Study population The study group consists of eleven WG patients who failed to achieve remission (defined as the absence of signs of new or worse disease activity, with persistent disease activity for $\leq 1$ item according to
Birmingham Vasculitis Activity Score for WG [BVAS/ WG] score) within 6 months despite chemotherapeutic treatment for life or vital organ threatening disease $[13,14]$. The patients were retrospectively recruited in 2009/2010. All patients fulfilled American College of Rheumatology classification criteria and Chapel Hill Consensus Conference definition for $\mathrm{WG}[15,16]$. Exclusion criteria included: a) limited WG or b) contraindication to CMR including MR-incompatible implants or electronic devices, renal insufficiency (creatinine clearance $<60 \mathrm{ml} / \mathrm{kg} / \mathrm{min}$ ), inability to perform breath hold and claustrophobia. Disease activity was assessed using BVAS/WG score [13]. Organ damage was measured using Vasculitis Damage Index (VDI), and disease extent was assessed by Disease Extent Index (DEI) [17, 18]. Sex- and age-matched healthy individuals without known inflammatory and cardiovascular diseases served as controls to compare findings of CMR. This study was approved by institutional ethics committee and complied with 1975 Declaration of Helsinki. Informed consent was obtained from each patient.

Combination of cyclophosphamide and glucocorticoids was used for remission induction. Cyclophosphamide was given intravenously at $15 \mathrm{mg} / \mathrm{kg}$ body weight ( $\max 1.2 \mathrm{~g}$ )
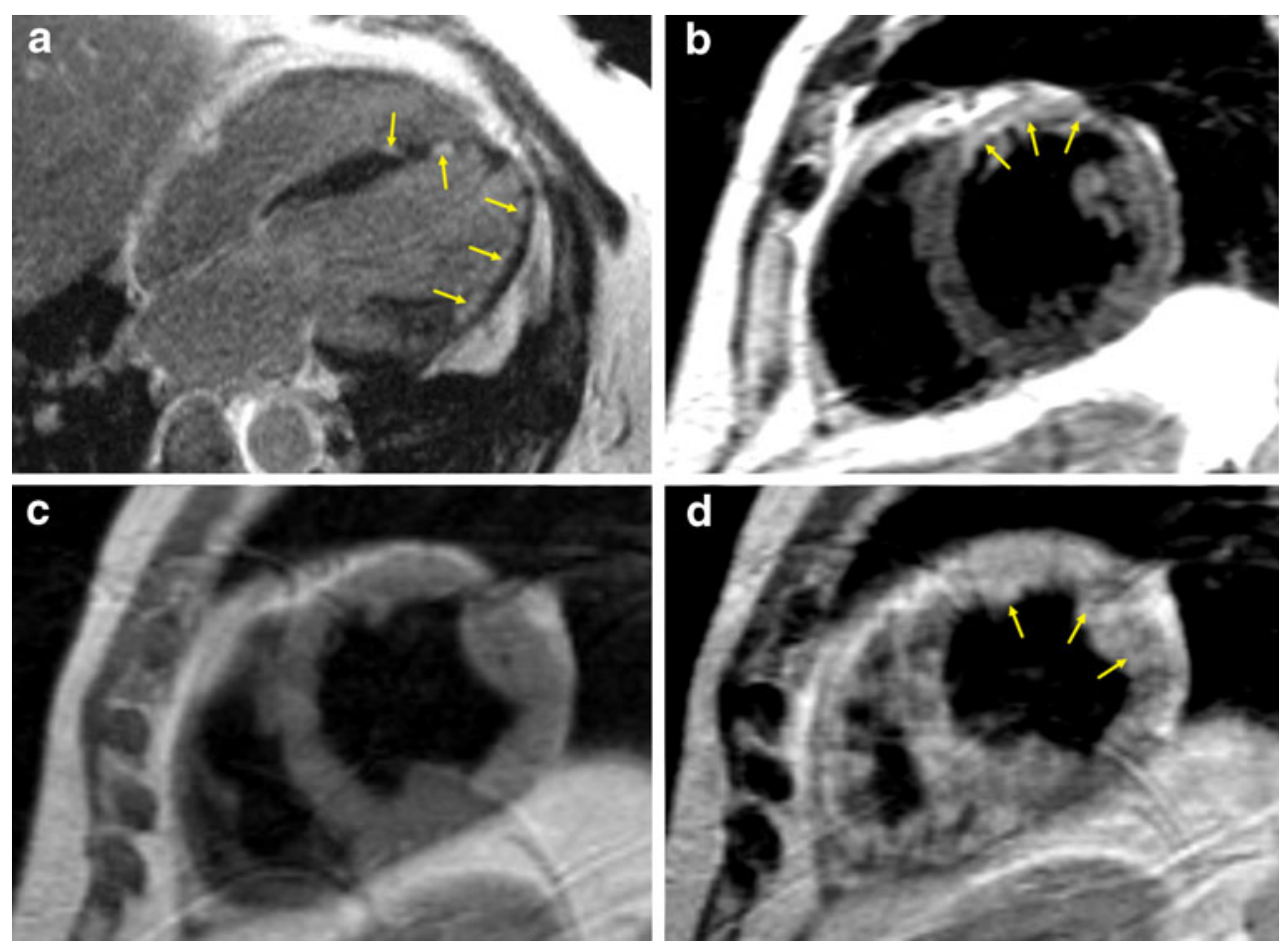

Fig. 1 Late gadolinium enhancement (LGE) image (a) in fourchamber apical view as well as TSE T2-weighted (b), pre- (c) and post-contrast TSE T1-weighted images (d) in short axis view in 58-years old male with Wegener's granulomatosis. LGE image shows subendocardial fibrosis in left and right ventricle (arrows) (a). Mid segments demonstrate increased T2 signal intensity (arrows) on TSE T2-weighted image (b) suggesting myocardial edema and early gadolinium enhancement (arrows) on post-contrast TSE T1-weighted image (d) reflecting increased myocardial hyperemia/capillary leak. Cardiac magnetic resonance revealed preserved global left ventricular systolic function $(\mathrm{EF}=56 \%)$ with regional wall motion abnormalities (akinesis of the apex, hypokinesis of apical anterior, lateral, septal and mid anteroseptal segments) 
every 4 weeks for 6 months [19]. Concomitant glucocorticoid therapy was initiated with intravenous methyloprednisolone (500-1000 $\mathrm{mg}$ ) for 3 consecutive days, followed by daily prednisone at $1 \mathrm{mg} / \mathrm{kg}$ body weight, which was subsequently tapered with the goal to reach $10 \mathrm{mg}$ /day [19]. As patients failed to achieve remission at 6 months, therapy was individualized and adapted to the disease extent and activity. This therapy included combination of low-dose glucocorticoids and either cyclophosphamide (up to $1.5 \mathrm{mg} / \mathrm{kg} /$ day), azathioprine (up to $2 \mathrm{mg} / \mathrm{kg}$ body weight) or methothrexate (up to $20-25 \mathrm{mg} / \mathrm{kg}$ body weight/week) with the goal to use the lowest effective dose and replace cyclophosphamide with azathioprine or methothrexate, if possible [12, 19]. Rituximab was not considered into drug regimen. All patients received prophylaxis against pneumocystis infection and osteoporosis [19].

\section{Cardiac magnetic resonance: imaging protocol}

Breath-hold, ECG gated CMR was performed at $1.5 \mathrm{~T}$ (Magnetom Sonata Maestro Class, Siemens, Erlangen, Germany) equipped with cardiac phased array coil. Cine and morphologic imaging was performed in left ventricular (LV) two-chamber, four-chamber and long-axis apical as well as in short-axis view encompassing the entire LV. Cine imaging was obtained using balanced steady-state free precession gradient echo technique (slice thickness $8 \mathrm{~mm}$, no gap, matrix $256 \times 192$, in-plane resolution $1.3 \times 1.3 \mathrm{~mm}$, TR/TE 39/1.1 ms, flip angle $59^{\circ}$ ). Morphologic images were acquired with body coil using TSE T2-weighted imaging (slice thickness $8 \mathrm{~mm}$, no gap, matrix $256 \times 154$, in-plane resolution $1.7 \times 1.3 \mathrm{~mm}$, TR depending upon RR interval, TE $78 \mathrm{~ms}$ ), and TSE T1-weighted imaging (slice thickness $8 \mathrm{~mm}$, no gap, TR depending upon RR interval, TE $6,9 \mathrm{~ms}$, flip angle $180^{\circ}$ ). TSE T1-weighted imaging was performed before and early after intravenous infusion of $0.1 \mathrm{mmol} / \mathrm{kg}$ body weight gadobutrol (Gadovist, Bayer Schering Pharma, Berlin, Germany). Following contrast medium injection $(10 \mathrm{~min})$, late gadolinium-enhanced (LGE) imaging was performed using T1-weighted segmented inversion-recovery pulse sequence (slice thickness $8 \mathrm{~mm}$, no gap, matrix $256 \times 192$, in-plane resolution $1.3 \times$ $1.3 \mathrm{~mm}, \mathrm{TR} / \mathrm{TE} 650 / 4.9 \mathrm{~ms}$, flip angle $30^{\circ}$, TI set to null normal myocardium).

\section{Transthoracic echocardiography}

Transthoracic echocardiography (TTE) was performed with Vivid 7 ultrasound systems (GE Vingmed Ultrasound A/S,
Horten, Norway). Analysis was performed off-line by an independent, experienced echocardiographer blinded to clinical and CMR data. Left ventricular (LV) ejection fraction (EF), end-diastolic volume (EDV) and end-systolic volume (ESV) were calculated. LV diastolic dysfunction was evaluated in accordance to guidelines published by the American Society of Echocardiography [20].

\section{Cardiac magnetic resonance: image analysis}

TSE T1-weighted before and after contrast medium, TSE T2-weighted, cine and LGE images were assessed off-line using dedicated software (MASS Medis, Leiden, the Nedtherlands) by two independent observers, blinded to clinical and echocardiographic data. Discrepancies in qualitative assessment were resolved by consensus. A seventeen segment LV model was used for analysis.

Cine images LV short-axis as well as two-chamber, fourchamber and long-axis apical views were used for segmental wall motion analysis. Segmental myocardial contractile function was scored as: 1-normal, 2-hypokinetic, 3-akinetic, 4-dyskinetic and expressed as wall motion score index (WMSI). Endocardial and epicardial borders were outlined on short axis images to calculate EDV, ESV, myocardial mass and ejection fraction (EF). EDV, ESV and myocardial mass were indexed to body surface area.

TSE T1- and T2-weighted images By quantitative analysis mean T2 and T1 signal intensity (SI) (pre- and postcontrast) were measured in a) individual LV segments, b) entire LV myocardium at mid LV level in short axis view and c) skeletal muscle (erector spinae or lattisimus dorsi) in the same slice. T2 myocardial SI was related to skeletal muscle SI and T2-SI ratio was calculated.. Myocardial early gadolinium enhancement (EGE) ratio was calculated as myocardial to skeletal muscle enhancement ratio. Global (i.e. determined in entire LV myocardium) T2-SI ratio $\geq 2.0$ and global EGE ratio $\geq 4$ were considered abnormal indicating myocardial edema and myocardial hyperaemia/hyperpermeability of capillaries, respectively [21]. By analogy, T2-SI ratio $\geq 2.0$ and EGE ratio $\geq 4$ were considered abnormal for individual $\mathrm{LV}$ segments.

$L G E$ images LGE images were assessed qualitatively for the presence and location of hyperintense lesions in contrast to hypointense viable myocardium. The number of affected segments was calculated and right ventricular (RV) involvement was determined. LGE lesions in LV myocardium were defined as transmural, subendocardial (adjacent to endocardium), midwall and subepicardial 
(adjacent to epicardium). Based on visual assessment of distribution LGE lesions were classified as non-ischaemic or ischaemic [21].

Myocardial inflammation According to Lake Louise Criteria CMR was considered to indicate myocardial inflammation, when $\geq 2$ of following criteria were present: a) global T2-SI ratio $\geq 2.0$, b) global EGE ratio $\geq 4$ and c) $\geq 1$ focal nonischaemic LGE lesion [21].

Statistical analysis Categorical data are presented as numbers or percentages, while continuous data as mean \pm standard deviation or median with interquartile range, where appropriate. The normal distribution was verified using Shapiro-Wilk test. Categorical variables were compared by Fisher's exact test, while continuous variables by unpaired student $t$-test or Wilcoxon rank-sum test. Pearson product-moment correlation was performed to assess relationship between the extent of LV damage and parameters reflecting WG activity. Statistical analyses were performed using SPSS software (version 12.0, SPSS Inc., Chicago, IL, USA). Differences were considered statistically significant at $p<0.05$.

\section{Results}

Baseline characteristics Eleven WG patients (eight males, three females, mean age $47 \pm 13$ years) underwent TTE and CMR successfully. Clinical characteristics including individual organ involvement are presented in Tables 1 and 2 . cANCA with anti-proteinase-3 specificity were found in all patients. Two patients had symptomatic heart failure (NYHA II and NYHA III), and one had typical stable angina (CCS III). All of them underwent coronary angiography which revealed no significant coronary artery stenoses. One patient had a history of prior myocardial infarction. No coronary artery revascularization was performed. At enrollment no patient showed AV-block on ECG although one demonstrated transient AV-block (grade III) during disease exacerbation.

CMR and TTE CMR characteristics are summarized in Table 3. No WG patient had global LV or RV systolic dysfunction, defined as LVEF $<50 \%$ and RVEF $<40 \%$, respectively. Both patients with heart failure symptoms demonstrated segmental LV wall motion abnormalities (apical hypokinesis [WMSI $=1.06]$ in one patient and akinesis of the apex with hypokinesis of the apical anterior, lateral and septal as well as mid anteroseptal segments [WMSI=1.35]) in another). According to Lake Louise Criteria CMR demonstrated myocardial inflammation in six WG patients. Pericardial effusion was found in five patients and localized pericardial thickening in six patients. The latter finding tended to be more frequent in those with than without CMR signs of myocarditis (5 vs $1 ; p=0.08$ ). Comparing patients with and without CMR signs of myocarditis, the former had more frequent diastolic dysfunction detected by TTE ( 5 vs $0, p=0.02$ ) and a higher index of LV mass $(72.6 \pm 9.4$ vs $57.8 \pm 7.7, p=0.02)$. No differences in the prevalence of hypertension ( 3 vs $3, p=$ 1.0 ) or diabetes mellitus ( 1 vs $1, p=1.0$ ) was observed between both groups. All individuals with LV diastolic dysfunction demonstrated an impaired relaxation pattern and none demonstrated a pseudonormal or restrictive diastolic dysfunction pattern. Comparing patients with or without CMR signs of myocarditis no differences in actual/ last flare DEI or BVAS/WG score were found and both groups showed similar cumulative VDI score at enrollment. LGE lesions were observed in LV or RV myocardium in nine and four patients, respectively. Patients with CMR signs of myocarditis had more LV segments with LGE than those without myocarditis (5.2 \pm 3.4 vs $1.0 \pm 1.2, p=0.03)$. LGE lesion were found in all LV myocardial fiber layers (5 subendocardium, 5 midwall and 4 supepicardium) and were transmural in two patients. Of $31 \mathrm{LV}$ segments with LGE lesions, 15 were localized in the inferior and posterior walls and 13 were apical. No correlation was found between the number of LV segments with LGE and actual/last flare DEI, BVAS/WG score, serum C-reactive protein, cANCA or cumulative VDI score $(r<0.4, p=\mathrm{NS}$ for all correlations). TTE demonstrated thickened mitral valve leaflets in six and thickened aortic valve leaflets in three patients. No patient demonstrated moderate or severe valvular regurgitation or stenosis.

\section{Discussion}

This is the first comprehensive evaluation of heart involvement in patients with life or organ threatening WG who were resistant to induction therapy. The heart was frequently involved and the majority of WG patients demonstrated LGE lesions distributed in all myocardial layers. Moreover, many patients showed signs of myocardial inflammation, which was associated with diastolic dysfunction.

Although clinical cardiac involvement is not common in WG, there is growing evidence of subclinical heart involvement during the course of this disease [2-5]. Previous studies showed the wide spectrum and varied prevalence of cardiac abnormalities in WG, which has been linked to the disease activity [3]. We found that heart involvement is very frequent in WG patients who despite $>6$ months induction therapy failed to achieve remission. In the majority of 


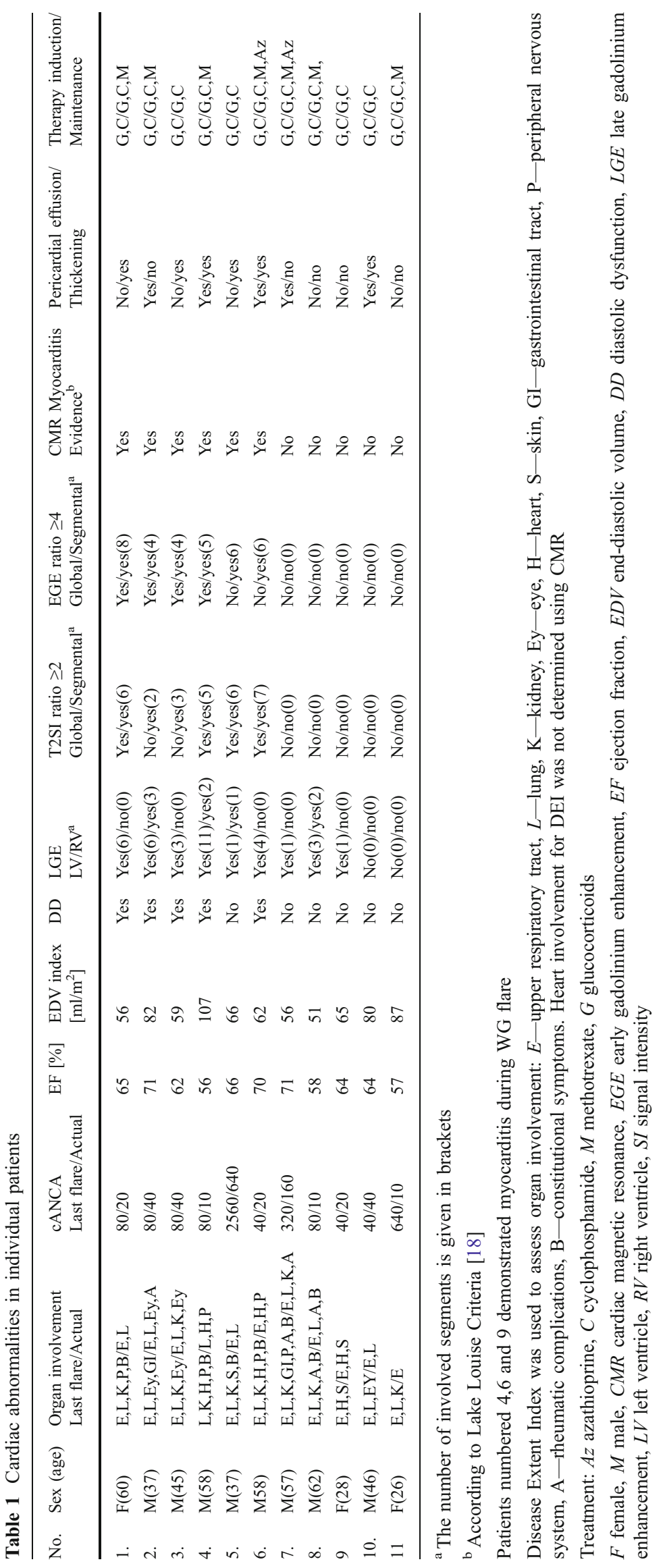


Table 2 Patient characteristics

\begin{tabular}{|c|c|c|}
\hline Parameters & $\begin{array}{l}\text { CMR evidence of myocarditis } \\
\text { Yes }(n=6) / \text { no }(n=5)\end{array}$ & All patients $(n=11)$ \\
\hline Female/Male & $1 / 5 / 2 / 3$ & $3 / 8$ \\
\hline Age [years] & $51.3 \pm 12.3 / 43.8 \pm 16.4$ & $46.7 \pm 13.2$ \\
\hline Time: diagnosis to CMR[months] & $55.5 \pm 34.8 / 66.0 \pm 63.3$ & $60.3 \pm 47.3$ \\
\hline Time: last flare to CMR [months] & $18.7 \pm 10.0 / 18.4 \pm 14.8$ & $18.5 \pm 11.7$ \\
\hline Time: end of induction therapy to CMR [months] & $12.7 \pm 10.0 / 12.4 \pm 14.8$ & $12.6 \pm 11.7$ \\
\hline \multicolumn{3}{|l|}{ Last flare } \\
\hline DEI & $9.0 \pm 1.1 / 8.0 \pm 3.1$ & $8.6 \pm 2.2$ \\
\hline BVAS/WG score & $12.5 \pm 3.4 / 12.5 \pm 4.0$ & $12.5 \pm 3.5$ \\
\hline cANCA titre & $80(80-80) / 80(40-400)$ & $80(50-260)$ \\
\hline $\mathrm{CRP}[\mathrm{mg} / \mathrm{l}]$ & $71 \pm 62 / 74 \pm 56$ & $72 \pm 57$ \\
\hline Creatinine clearance $[\mathrm{ml} / \mathrm{min}]$ & $97 \pm 49 / 87 \pm 40$ & $93 \pm 43$ \\
\hline \multicolumn{3}{|l|}{ Enrolment } \\
\hline DEI & $6.0 \pm 1.8 / 5.2 \pm 2.2$ & $5.6 \pm 1.9$ \\
\hline BVAS/WG score & $4.2 \pm 1.5 / 4.6 \pm 1.7$ & $4.4 \pm 1.5$ \\
\hline Cumulative VDI score & $7.8 \pm 0.8 / 7.2 \pm 2.6$ & $7.5 \pm 1.8$ \\
\hline cANCA titre & $30(20-40) / 20(10-70)$ & $20(12.5-40)$ \\
\hline $\mathrm{CRP}[\mathrm{mg} / \mathrm{l}]$ & $6.9 \pm 6.6 / 7.4 \pm 7.4$ & $7.1 \pm 6.6$ \\
\hline Creatinine clearance $[\mathrm{ml} / \mathrm{min}]$ & $90 \pm 48 / 105 \pm 34$ & $97 \pm 41$ \\
\hline Exertional dyspnea & $1 / 1$ & 2 \\
\hline NYHA class I/II/III/IV & $0 / 1 / 0 / 0 / 0 / 0 / 1 / 0$ & $0 / 1 / 1 / 0$ \\
\hline Angina pectoris & $0 / 1$ & 1 \\
\hline CCS class $1 / 2 / 3 / 4$ & $0 / 0 / 0 / 0 / 0 / 0 / 1 / 0$ & $0 / 0 / 1 / 0$ \\
\hline
\end{tabular}

No statistical differences between patients with and without CMR signs of myocarditis were found

$C M R$ cardiac magnetic resonance, BVAS/WG Birmingham Vasculitis Activity Score for Wegener's granulomatosis, CCS Canadian Cardiovascular Society classification system for angina pectoris, $c A N C A$ cytoplasmic pattern of antineutrophil cytoplasmic antibodies, $C R P C$-reacitive protein, $D E I$ Disease Extent Index, NYHA New York Heart Association classification system for heart failure, VDI Vasculitis Damage Index

patients cardiac involvement was subclinical and only two patients manifested cardiac symptoms (heart failure) at enrollment. The most frequent abnormality observed in the present study was LGE lesions in both RV and LV myocardium. LGE lesions were previously described in case reports of WG and similar to the present study, they were distributed in all myocardial layers (subendocardial, midwall, subepicardial) [9-11]. Interestingly, many of them were localized in apical and posteroinferior LV wall segments and a similar distribution of myocarditis was reported at autopsy in a patient with rapidly progressive, fatal WG [22]. However, other distributions of myocardial involvement were also described [9-11]. In contrast to the present study, Mavrogeni et al. [4], who performed CMR in eleven WG patients, did not observe LGE myocardial lesions. This apparent discrepancy may have several explanations. First, WG patients described by Mavrogeni et al. [4] had less active disease. As such, they may have not developed necrotic/fibrotic myocardial lesions or such lesions may have resolved during the course of well controlled disease. Indeed, regression of LGE lesions were reported by Mahrhold et al.
[23] following viral myocarditis. Second, patients enrolled by Mavrogeni et al. [4] had no history or evidence of cardiac disease (versus four such patients in the current series). Third, the high threshold for signal intensity to delineate LGE lesions used by Mavrogeni et al. [4] may have reduced the ability to detect such lesions. Prior studies which evaluated non-ischemic myocardial damage used lower thresholds for LGE detection. [23, 24] In the current study, LGE lesions were more frequent in patients with CMR signs of myocardial inflammation. Nevertheless, these lesions were frequently located in LV myocardial segments without CMR signs of myocardial oedema or hyperaemia/hyperpermeability of capillaries suggesting rather healed than necrotic lesions. In the majority of patients LGE lesions were not associated with regional wall motion abnormalities. In this regard, Mahrhold et al. [25] demonstrated that nontransmural or transmural of low volume LGE lesions following myocardial infarction rarely cause contractile abnormalities.

The assessment of WG disease activity is crucial to guide treatment and assess prognosis. Our study is the 
Table 3 CMR findings

\begin{tabular}{lll}
\hline CMR Parameters & $\begin{array}{l}\text { CMR evidence of myocarditis in WG } \\
\text { Yes }(n=6) / \text { no }(n=5)\end{array}$ & $\begin{array}{l}\text { WG patients }(n=11) / \text { Matched } \\
\text { controls }(n=11)\end{array}$ \\
\hline LVEF [\%] & $65.0 \pm 5.5 / 62.8 \pm 5.6$ & $64.0 \pm 5.4 / 61.4 \pm 3.2$ \\
LVEDV index $\left[\mathrm{ml} / \mathrm{m}^{2}\right]$ & $72.0 \pm 19.5 / 67.8 \pm 15.4$ & $70.1 \pm 17.0 / 72.6 \pm 12.8$ \\
LVMass index $\left[\mathrm{g} / \mathrm{m}^{2}\right]$ & $72.6 \pm 9.4 / 57.8 \pm 7.7$ & $65.9 \pm 11.3 / 58.1 \pm 9.0$ \\
RVEF [\%] & $59.7 \pm 6.1 / 50.6 \pm 10.6$ & $55.6 \pm 9.3 / 52.7 \pm 6.6$ \\
RVEDV index $\left[\mathrm{ml} / \mathrm{m}^{2}\right]$ & $70.9 \pm 13.8 / 71.8 \pm 23.0$ & $71.3 \pm 17.5 / 73.2 \pm 9.1$ \\
RVMass index $\left[\mathrm{g} / \mathrm{m}^{2}\right]$ & $17.5 \pm 3.6 / 14.9 \pm 4.3$ & $16.7 \pm 2.7 / 16.3 \pm 3.9$ \\
Myocardial SI-T2 ratio at mid LV level & $1.8 \pm 0.5 / 1.3 \pm 0.1$ & $1.5 \pm 0.5 / 0.7 \pm 0.4 * * * *$ \\
Myocardial EGE ratio at mid LV level & $4.1 \pm 0.5 / 2.3 \pm 0.5^{* *}$ & $3.3 \pm 1.0 / 2.3 \pm 0.9 * * *$ \\
Pericardial effusion & $3 / 2$ & $5 / 0^{* * *}$ \\
Localized pericardial thickening & $5 / 1$ & $6 / 0^{* * *}$ \\
LVLGE: present & $6 / 3$ & $9 / 0^{* * * *}$ \\
LVLGE: segment number & $5.2 \pm 3.4 / 1.0 \pm 1.2^{*}$ & $3.3 \pm 3.3 / 0.0 \pm 0.0^{* * * *}$ \\
RVLGE: present & $3 / 1$ & $4 / 0$ \\
RVLGE: segment number & $1.0 \pm 1.3 / 0.4 \pm 0.9$ & $0.7 \pm 1.1 / 0.0 \pm 0.0^{* * *}$ \\
\hline
\end{tabular}

${ }^{*} p<0.05, * * p<0.001$ for patients with versus without CMR signs of myocarditis

$* * * p<0.05, * * * * p<0.001$ for WG patients versus controls

$C M R$ cardiac magnetic resonance, $E G E$ early gadolinium enhancement, $E F$ ejection fraction, $E D V$ end-diastolic volume, $L G E$ late gadolinium enhancement, $L V$ left ventricular, $R V$ right ventricular, $W G$ Wegener's granulomatosis

first to demonstrate that CMR signs of myocarditis are frequent in patients with WG who fail to achieve remission despite $>6$ months induction therapy. If compared to our study, prior reports suggested lower prevalence of myocarditis in WG [26]. The high prevalence of myocardial inflammation may reflect increased disease activity and/or high disease affinity for heart in those who are resistant to induction therapy. This may be clinically pertinent since heart involvement is recognized as a factor predicting relapse [27]. The signs suggestive of myocarditis were accompanied by higher prevalence of LGE lesions and localized pericardial thickening indicating more severe and extensive inflammatory process. Clinical and prognostic implications of CMR findings in WG are unknown and their responsiveness to immunosuppressive therapy remains to be determined. Interestingly, Caudron $\mathrm{J}$ et al. [10] previously demonstrated regression of CMR detected myopericarditis after 3 months treatment with corticosteroids and cyclophosphamide. Similarly, regression of CMR-diagnosed myocarditis following rituximab therapy, which was administered when conventional regimens failed to control disease, were described [9]. In the present study, the majority of patients with CMR evidence of active myocarditis demonstrated diastolic dysfunction on TTE, which may reflect increased LV wall stiffness. Patients with CMR signs of myocarditis had a larger LV mass index which could be partially explained by the presence of myocardial edema although hypertrophy secondary to hypertension cannot be excluded. As diastolic dysfunction was present in the majority of patients with active myocarditis, TTE may serve as a screening tool before CMR is performed.

Study limitations First, the number of enrolled patients is low and the results should be confirmed in larger patient cohort. Second, due to contraindication to CMR we excluded patients with low creatinine clearance so the results may not apply to the larger population of WG patients. Third, CMR criteria recommended to identity myocardial inflammation are based on studies over suspected acute or chronic viral myocarditis, and were not validated when inflammation is caused by autoimmune process. Finally, endomyocardial biopsy was not performed to confirm the presence of myocarditis. It is known, however, that endomyocardial biopsy has a poor diagnostic accuracy due to patchy myocardial involvement in WG [22].

\section{Conclusions}

Cardiac involvement is frequent in patients with life or organ threatening WG who fail to achieve remission despite $>6$ months induction therapy. Most patients demonstrate LGE lesions, which may reflect both necrotic and fibrotic lesions. Moreover, heart disease is 
frequently characterized by myocardial inflammatory process. As myocardial inflammation was frequently associated with diastolic dysfunction on TTE, this modality may serve as a screening tool for myocarditis in WG. The present study suggests that CMR appears to be the most sensitive non-invasive diagnostic technique to assess heart involvement in WG.

Open Access This article is distributed under the terms of the Creative Commons Attribution Noncommercial License which permits any noncommercial use, distribution, and reproduction in any medium, provided the original author(s) and source are credited.

\section{References}

1. Hoffman GS, Kerr GS, Leavitt RY, Hallahan CW, Lebovics RS, Travis WD, Rottem M, Fauci AS (1992) Wegener granulomatosis: an analysis of 158 patients. Ann Intern Med 116:488-498

2. Morelli S, Gurgo Di Castelmenardo AM, Conti F, Sgreccia A, Alessandri C, Bernardo ML, Valesini G (2000) Cardiac involvement in patients with Wegener's granulomatosis. Rheumatol Int 19:209-212

3. Knockaert DC (2007) Cardiac involvement in systemic inflammatory diseases. Eur Heart J 28:1797-1804

4. Mavrogeni S, Manoussakis MN, Karagiorga TC, Douskou M, Panagiotakos D, Bournia V, Cokkinos DV, Moutsopoulos HM (2009) Detection of coronary artery lesions and myocardial necrosis by magnetic resonance in systemic necrotizing vasculitides. Arthritis Rheum 61:1121-1129

5. Oliveira GH, Seward JB, Tsang TS, Specks U (2005) Echocardiographic findings in patients with Wegener granulomatosis. Mayo Clin Proc 80:1435-1440

6. Abdel-Aty H, Boyé P, Zagrosek A, Wassmuth R, Kumar A, Messroghli D, Bock P, Dietz R, Friedrich MG, Schulz-Menger J (2005) Diagnostic performance of cardiovascular magnetic resonance in patients with suspected acute myocarditis: comparison of different approaches. J Am Coll Cardiol 45:1815-1822

7. Röttgen R, Christiani R, Freyhardt P, Gutberlet M, Schultheiss HP, Hamm B, Kühl U (2011) Magnetic resonance imaging findings in acute myocarditis and correlation with immunohistological parameters. Eur Radiol 21:1259-1266

8. Gutberlet M, Spors B, Thoma T, Bertram H, Denecke T, Felix R, Noutsias M, Schultheiss HP, Kühl U (2008) Suspected chronic myocarditis at cardiac MR: diagnostic accuracy and association with immunohistologically detected inflammation and viral persistence. Radiology 246:401-409

9. Brihaye B, Aouba A, Pagnoux C, Vignaux O, Le Hello C, Guillevin L (2008) Rituximab reversed cardiac involvement of Wegener's granulomatosis: magnetic resonance imaging assessment. Presse Med 37:412-415

10. Caudron J, Fares J, Dominique S, Dacher JN (2009) Diagnosis and follow-up of Wegener's granulomatosis by cardiac magnetic resonance. Eur Heart J 30:1537

11. To A, De Zoysa J, Christiansen JP (2007) Cardiomyopathy associated with Wegener's granulomatosis. Heart 93:984

12. Langford CA (2007) Small-vessel vasculitis: therapeutic management. Curr Rheumatol Rep 9:328-335

13. Stone JH, Hoffman GS, Merkel PA, Min YI, Uhlfelder ML, Hellmann DB, Specks U, Allen NB, Davis JC, Spiera RF, Calabrese LH, Wigley FM, Maiden N, Valente RM, Niles JL, Fye KH, McCune JW, St Clair EW, Luqmani RA (2001) A disease-specific activity index for Wegener's granulomatosis: modification of the Birmingham Vasculitis Activity Score. Arthritis Rheum 44:912-920

14. Jayne D, Rasmussen N, Andrassy K, Bacon P, Tervaert JW, Dadoniené J, Ekstrand A, Gaskin G, Gregorini G, de Groot K, Gross W, Hagen EC, Mirapeix E, Pettersson E, Siegert C, Sinico A, Tesar V, Westman K, Pusey C, European Vasculitis Study Group (2003) A randomized trial of maintenance therapy for vasculitis associated with antineutrophil cytoplasmic autoantibodies. N Engl J Med 349:36-44

15. Leavitt RY, Fauci AS, Bloch DA, Michel BA, Hunder GG, Arend WP, Calabrese LH, Fries JF, Lie JT, Lightfoot RW Jr (1990) The American College of Rheumatology 1990 criteria for the classification of Wegener's granulomatosis. Arthritis Rheum 33:1101-1107

16. Jennette JC, Falk RJ, Andrassy K, Bacon PA, Churg J, Gross WL, Hagen EC, Hoffman GS, Hunder GG, Kallenberg CG (1994) Nomenclature of systemic vasculitides. Proposal of an international consensus conference. Arthritis Rheum 37:187-192

17. Exley AR, Bacon PA, Luqmani RA, Kitas GD, Gordon C, Savage CO, Adu D (1997) Development and initial validation of the Vasculitis Damage Index for the standardized clinical assessment of damage in the systemic vasculitides. Arthritis Rheum 40:371-380

18. de Groot K, Gross WL, Herlyn K, Reinhold-Keller E (2001) Development and validation of a disease extent index for Wegener's granulomatosis. Clin Nephrol 55:31-38

19. Mukhtyar C, Guillevin L, Cid MC, Dasgupta B, de Groot K, Gross W, Hauser T, Hellmich B, Jayne D, Kallenberg CG, Merkel PA, Raspe H, Salvarani C, Scott DG, Stegeman C, Watts R, Westman K, Witter J, Yazici H, Luqmani R (2009) EULAR recommendations for the management of primary small and medium vessel vasculitis. Ann Rheum Dis 68:310-317

20. Nagueh SF, Appleton CP, Gillebert TC, Marino PN, Oh JK, Smiseth OA, Waggoner AD, Flachskampf FA, Pellikka PA, Evangelista A (2009) Recommendations for the evaluation of left ventricular diastolic function by echocardiography. J Am Soc Echocardiogr 22:107-133

21. Friedrich MG, Sechtem U, Schulz-Menger J, Holmvang G, Alakija P, Cooper LT, White JA, Abdel-Aty H, Gutberlet M, Prasad S, Aletras A, Laissy JP, Paterson I, Filipchuk NG, Kumar A, Pauschinger M, Liu P (2009) International Consensus Group on Cardiovascular Magnetic Resonance in Myocarditis. Cardiovascular magnetic resonance in myocarditis: a JACC White Paper. J Am Coll Cardiol 53:1475-1487

22. Mukhopadhyay S, Hensley RG, Tazelaar HD (2010) Cardiac involvement in Wegener granulomatosis diagnosed at autopsy. Cardiovasc Pathol 19:312-315

23. Mahrholdt H, Wagner A, Deluigi CC, Kispert E, Hager S, Meinhardt G, Vogelsberg H, Fritz P, Dippon J, Bock CT, Klingel K, Kandolf R, Sechtem U (2006) Presentation, patterns of myocardial damage, and clinical course of viral myocarditis. Circulation 114:1581-1590

24. Assomull RG, Prasad SK, Lyne J, Smith G, Burman ED, Khan M, Sheppard MN, Poole-Wilson PA, Pennell DJ (2006) Cardiovascular magnetic resonance, fibrosis, and prognosis in dilated cardiomyopathy. J Am Coll Cardiol 48:1977-1985

25. Mahrholdt H, Wagner A, Parker M, Regenfus M, Fieno DS, Bonow RO, Kim RJ, Judd RM (2003) Relationship of contractile function to transmural extent of infarction in patients with chronic coronary artery disease. J Am Coll Cardiol 42:505-512

26. Forstot JZ, Overlie PA, Neufeld GK, Harmon CE, Forstot SL (1980) Cardiac complications of Wegener granulomatosis: a case report of complete heart block and review of the literature. Semin Arthritis Rheum 10:148-154

27. Mukhtyar C, Flossmann O, Hellmich B, Bacon P, Cid M, CohenTervaert JW, Gross WL, Guillevin L, Jayne D, Mahr A, Merkel PA, Raspe H, Scott D, Witter J, Yazici H, Luqmani RA, European Vasculitis Study Group (EUVAS) (2008) Outcomes from studies of antineutrophil cytoplasm antibody associated vasculitis: a systematic review by the European League Against Rheumatism systemic vasculitis task force. Ann Rheum Dis 67:1004-1010 